\title{
Influence of testicular fluid infected with Treponema pallidum on intradermal lesions
}

\author{
T J FITZGERALD AND R C JOHNSON \\ From the Department of Medical Microbiology, University of Minnesota Medical School, Minneapolis, \\ Minnesota, USA
}

SUMMARY A viscous mucoid fluid occasionally accumulates after intratesticular inoculation of rabbits with Treponema pallidum. Experiments were performed to assess the effects of this testicular fluid on the development of syphilitic lesions. Intramuscular injections of this fluid altered host defences as indicated by shorter incubation periods, by reactivation of healing lesions, and by the presence of lesions at a time when solid immunity should have developed.

\section{Introduction}

Mucoid material characteristically accumulates within syphilitic lesions. This material contains acidic mucopolysaccharides as indicated by metachromasia. ${ }^{1-4}$ These mucopolysaccharides have been tentatively identified as hyaluronic acid and chondroitin sulphate based on their partial degradation by hyaluronidase. ${ }^{134}$ Treponema pallidum (Nichols strain) has an outer layer of amorphous material composed of mucopolysaccharide as shown by reaction with ruthenium red, toluidine blue, acidified bovine serum albumin, wheat-germ agglutinin, and soybean agglutinin..$^{5-7}$ Fitzgerald and Johnson $^{6}$ suggested that the mucoid material within lesions results from treponemal biosynthesis of this outer mucopolysaccharide layer.

The effects of the mucoid testicular fluid on syphilitic lesions in rabbits were examined with an unexpected result.

\section{Material and methods}

The Nichols strain of $T$ pallidum was maintained by intratesticular passage in Dutch Belt rabbits. Each testis was inoculated with $10^{7}$ to $3 \times 10^{7}$ treponemes. Within 9-14 days the animals were killed and the organisms were harvested as previously described. ${ }^{8}$

After intratesticular inoculation, a mucoid fluid accumulated within the membranes of some of the infected testes. This testicular fluid (TF) was withdrawn by needle aspiration and $15 \mathrm{mmol} / \mathrm{l}$

Address for reprints: Dr T J Fitzgerald, Department of Medical Microbiology, University of Minnesota, 2205 East Fifth Street, Duluth, Minnesota 55812, USA

Received for publication 2 October 1978 dithiothreitol was added. The mucoid TF contained numerous actively motile treponemes. Heating of this preparation for $5-7$ minutes at $55^{\circ} \mathrm{C}$ inactivated the motility of the treponemes. This heated preparation was injected intradermally into rabbits to assess virulence. After 60 days no lesions were apparent, indicating that the heating process effectively killed the organisms.

The quantity of acidic mucopolysaccharide within the TF was determined by reaction with acidified bovine serum albumin. ${ }^{9}$ Each preparation of TF contained $5-10 \mathrm{mg}$ mucopolysaccharide $/ \mathrm{ml}$. One millilitre of heated TF was mixed vigorously with $1 \mathrm{ml}$ incomplete Freund's adjuvant (Difco). This suspension was then injected intramuscularly; each injection contained a total of $5-10 \mathrm{mg}$ mucopolysaccharide. All injections of TF were given intramuscularly in the lower portion of the back legs of the animals. All challenges with freshly harvested preparations of $T$ pallidum were injected intradermally on top of the backs of the animals. In separate experiments, the injection of incomplete Freund's adjuvant or the injection of extracts of uninfected rabbit testes did not affect the development of syphilitic dermal lesions.

\section{Results}

The effects of TF on syphilitic lesions in three related experiments are summarised in the table.

\section{FIRST EXPERIMENT}

In the first experiment, a rabbit was injected intramuscularly with TF containing 5-10 mg mucopolysaccharide. Four weeks later there was no evidence of treponemal lesions at the site of intramuscular injection or at any other site. At this 
TABLE Decreased incubation periods after intramuscular injection of infected testicular fluid (TF)

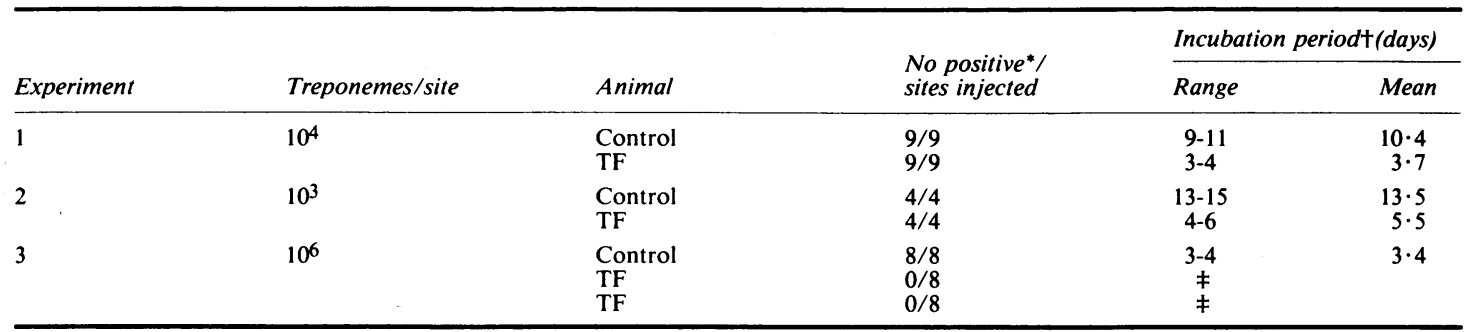

*Development of treponemal skin lesions

tInitial detection of erythema and induration

$\neq$ No treponemal skin lesions apparent after 60 days

time, this animal and a control animal not injected with TF were inoculated intradermally at nine sites on the shaved back with approximately $10^{4}$ treponemes/site. The incubation period before the appearance of erythema and induration was 9-11 days for the nine sites on the control animal. In the test animal, the incubation period was considerably shortened; all nine sites showed erythema and induration on days 3 and 4 . In our laboratory an incubation period of this duration usually occurs after inoculation of approximately $10^{6}$ treponemes/site. In this experiment $10^{4}$ treponemes/site were inoculated.

\section{SECOND EXPERIMENT}

\section{First inoculation}

In the second experiment, one rabbit was injected intramuscularly with TF containing 5-10 mg mucopolysaccharide. Four weeks later, this animal and a control animal were challenged intradermally at four sites with approximately $10^{3}$ treponemes $/ \mathrm{ml}$. As in the previous experiment, a shortening of the incubation period was observed in the TF-injected rabbit. In the control animal the mean incubation period was 13.5 days; in the test animal, it was $5 \cdot 5$ days. An incubation period of 5-6 days usually occurs after inoculation of approximately $10^{5}$ treponemes/site; in this experiment $10^{3}$ treponemes/site were inoculated

Aspirates of lesion material from each site were examined for the presence of actively motile treponemes. The four control and the four test sites gave positive results, containing 1-5 organisms per microscopic field at $\times \mathbf{4 3 0}$ magnification.

\section{Development of lesions}

As shown in the figure, the development of lesions in each animal progressed in typical fashion and within the expected time periods. Lesions were initially erythematous and slightly indurated; they increased in size and ulcerated within about 10 days. After two weeks the size and severity of ulceration were

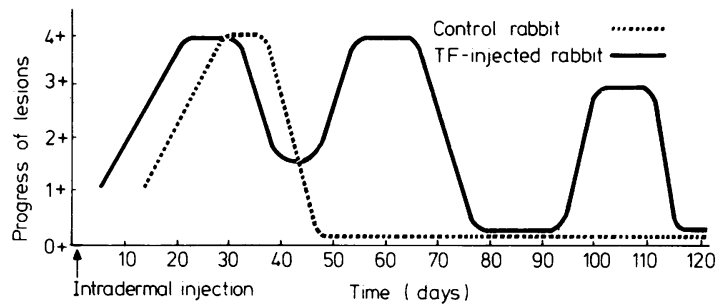

FIGURE Development of lesions in two rabbits inoculated intradermally on day 0 with $1 \times 10^{3}$ treponemes per site. Preparations of heated testicular fluid (TF) mixed with incomplete Freund's adjuvant were injected intramuscularly into one of the animals 30 days before day 0 and on day 36 and day 84. Development of lesions was rated as: $0+$, no symptoms; $1+$, initial erythema plus induration; $2+$, increased size plus erythema and induration; $3+$, beginning of ulceration; $4+$, ulceration; and $0+$, healed.

maximal. The lesions then began to heal. The only observable difference was the shorter incubation period in the TF-injected animal.

\section{Second inoculation}

A second preparation of TF containing $5-10 \mathrm{mg}$ mucopolysaccharide was injected intramuscularly into the test animal on day 36. All four dermal lesions continued to heal for approximately eight days. At this point, the healing process was interrupted. The four healing lesions began to increase in size and induration (figure). Darkfield samples of lesion material were examined on day 50 . Each of the four samples contained relatively large numbers of actively motile treponemes (10-50 organisms per microscopic field at $\times 430$ magnification).

The four lesions continued to increase in size and severity and again ulcerated. Healing was detectable within a few weeks. The development and time sequences of these lesions were typical for dermal lesions induced by $T$ pallidum. 


\section{Third inoculation}

On day 84, approximately 20 days after healing of the reactivated dermal lesions, a third preparation of TF containing 5-10 mg mucopolysaccharide was injected intramuscularly into the test animal. Within 10 days, three of the four previously healed lesions began to increase in size and induration (figure). Darkfield samples removed on day 105 showed sluggishly motile treponemes within three reactivated sites, but in this case far fewer treponemes were detected (one organism per 10 microscopic fields at $\times 430$ magnification).

The reactivation of these lesions was not as dramatic as that observed after the second injection of TF on day 36. The lesions were not as pronounced in size and severity, although they did ulcerate and healing occurred after a shorter interval.

Further attempts to reactivate the four dermal lesions by injecting preparations of TF intramuscularly on day 106 and day 122 were unsuccessful.

\section{THIRD EXPERIMENT}

In the third experiment, two immune rabbits were injected intramuscularly with TF containing 5-10 mg mucopolysaccharide. One animal had been injected intratesticularly with $6 \times 10^{7}$ treponemes three months previously and an obvious orchitis had developed; the second animal had been injected intratesticularly with $6 \times 10^{7}$ treponemes one year previously and had subsequently been challenged eight times with approximately $10^{8}$ treponemes per site at eight sites. Lesions developed in the control animal at all sites on days 3 and 4 . In contrast, no lesions were detected after 60 days in either immune animal. Thus, injections of TF in these experiments did not interfere with solid immunity after it had developed in syphilitic rabbits.

\section{Discussion}

The interruption of the healing process resulting in reactivation of dermal lesions was most unusual. There is only one other report of reactivation of syphilitic lesions and that involved antibiotic treatment. ${ }^{10}$ In experimental syphilis, once lesions begin to heal the healing continues. As shown in the figure, the four lesions on the control animal were completely healed by day 45 . Occasionally, disseminated syphilis in rabbits is observed. These disseminated lesions, however, occur at sites other than those initially inoculated intradermally. The reactivation of lesions after intramuscular injection of TF indicates that infectious treponemes were still present within the healing (day 36) and healed (day 84) lesions. TF in some way altered the usual host defences that develop in experimental syphilis, as indicated by shorter incubation periods and by reactivation of lesions. In addition, in this animal lesions were present at a time when solid immunity should have developed (day 90). Apparently, the interference with host defences resulted in re-establishment of the infectious process. Darkfield samples of the reactivated lesions contained motile treponemes, showing that these lesions were due to multiplication of $T$ pallidum.

A number of reports ${ }^{11-16}$ have speculated that immunosuppression occurs at certain stages of syphilitic infection. This was suggested by depletion of lymphoid cells within lymph nodes and spleens and by depression of cell-mediated immune responses. In the preliminary experiments reported in this paper, no attempt was made to determine the specific component within TF that was responsible for interfering with host defence mechanisms. TF is a complex mixture of both treponemal and host products and it contains large amounts of mucopolysaccharides. $T$ pallidum (Nichols strain) has an outer coat of mucopolysaccharide. ${ }^{5-7}$ Fitzgerald and Johnson $^{6}$ suggested that the mucoid material that accumulates during syphilitic infection results from treponemal biosynthesis of its mucopolysaccharide capsule and that the mucopolysaccharide within TF is primarily of treponemal origin. The compromise of host defences after injection of TF may reflect a type of immunosuppression. If this is the case, then the treponemal mucopolysaccharides might be immunosuppressive in syphilis. This interesting possibility may have direct application to the exacerbation of syphilis that results in the secondary stages of the disease.

Subsequent experiments strongly support the possibility that mucopolysaccharide material is immunosuppressive. ${ }^{17}$ TF inhibited the concanavalin-A-induced blast transformation of rabbit lymphocytes. Importantly, the inhibitory effect was destroyed by pretreatment of TF with a commercial preparation of hyaluronidase. This enzyme degrades mucopolysaccharides. In addition, syphilitic rabbit serum inhibited blast transformation. This serum contained mucopolysaccharides, presumably of treponemal origin. ${ }^{18}$ Pretreatment of this serum also destroyed the serum inhibitory effect on blast transformation.

This investigation was supported by Public Health Service grants AI-08124 and AI-12978 from the National Institutes of Allergy and Infectious Diseases. We wish to acknowledge the valuable technical assistance of Elizabeth Thompson Wolff.

\section{References}

1. Scott V, Dammin GJ. Hyaluronidase and experimental syphilis. III Metachromasia in syphilitic orchitis and its relation to hyaluronic acid. Amer J Syph 1950;34:501-14. 
2. Turner TB, Hollander DH. Cortisone in experimental syphilis. Johns Hopkins Hosp Bull 1950;87:505-9.

3. Scott V, Dammin GJ. Morphologic and histochemical sequences in syphilitic and in tuberculous orchitis in the rabbit. Amer J Syph 1954;38: 189-202.

4. Turner TB, Hollander DH. Studies on the mechanism of action of cortisone in experimental syphilis. Amer $J$ Syph 1954;38:371-87.

5. Fitzgerald TJ, Cleveland P, Johnson RC, Miller JN, Sykes JA. Scanning electron microscopy of Treponema pallidum (Nichols strain) attached to cultured mammalian cells. J Bacteriol 1976; 130: 1333-44.

6. Fitzgerald TJ, Johnson RC. Surface mucopolysaccharides of Treponema pallidum. Infect Immun 1979; 24:244-51.

7. Zeigler JA, Jones AM, Jones RH, Kubica KM. Demonstration of extracellular material at the surface of pathogenic $T$ pallidum cells. Br J Vener Dis 1976;52:1-8.

8. Fitzgerald TJ, Johnson RC, Miller JN, Sykes JA. Characterization of the attachment of Treponema pallidum (Nichols strain) to cultured mammalian cells and the potential relationship of attachment to pathogenicity. Infect Immun 1977; 18:467-78.

9. Ibrahim AN, Streitfeld MM. The microassay of hyaluronic acid concentration and hyaluronidase activity by capillary turbidity (CT) and capillary turbidity reduction (CTR) tests. Ann Biochem 1973; 56:428-34.
10. Ito K. A model of experimental syphilitic re-induratio. Euph Cacoph 1977;4: 16-40.

11. Metzger $\mathbf{M}$. The role of immunologic responses in protection against syphilis. In: Johnson RC, ed. The Biology of Parasitic Spirochetes. New York: Academic Press, 1976;327-37.

12. Metzger M, Podwinska J, Smogor W. Cell-mediated immunity in experimental syphilis in rabbits. Arch Immunol Ther Exp 1977; 25:25-34.

13. Levene GM, Turk JL, Wright DHM, Grimble AGS. Reduced lymphocyte transformation due to a plasma factor in patients with active syphilis. Lancet 1969;ii:246-7.

14. Wright DHM, Grimble AS. Why is the infectious stage of syphilis prolonged? $\mathrm{Br} J$ Vener Dis 1974; 50:45-9.

15. Musher DM, Schell RF, Knox JM. The immunology of syphilis. Int J Derm 1976; 15:566-76.

16. Wicher $\mathrm{V}$, Wicher $\mathrm{K}$. In-vitro cell response of Treponema pallidum-infected rabbits. Clin Exp Immunol 1977;24: 480-500.

17. Bey RF, Johnson RC, Fitzgerald, TJ. Suppression of lymphocyte response to concanavalin A by mucopolysaccharide material from Treponema pallidum-infected rabbits. Infect Immun 1979; 26:64-9.

18. Fitzgerald TJ, Johnson RC. Mucopolysaccharidase of Treponema pallidum. Infect Immun 1979;24:261-8. 